\title{
Genetic Analysis of Victorin Sensitivity and Identification of a Causal Nucleotide-Binding Site Leucine-Rich Repeat Gene in Phaseolus vulgaris
}

\author{
J. M. Lorang, ${ }^{1}$ C. H. Hagerty, ${ }^{2}$ R. Lee, ${ }^{3}$ P. E. McClean, ${ }^{3}$ and T. J. Wolpert ${ }^{1,+}$ \\ ${ }^{1}$ Department of Botany and Plant Pathology and Genome Research and Biocomputing, Oregon State University, Corvallis, OR \\ 97331-2902, U.S.A.; ${ }^{2}$ Columbia Basin Agricultural Research Center, Oregon State University, Adams, OR 97810, U.S.A.; and \\ ${ }^{3}$ Department of Plant Sciences and Genomics and Bioinformatics Program, North Dakota State University, Fargo, ND 58108- \\ 6050, U.S.A.
}

Accepted 23 April 2018.

\begin{abstract}
Cochliobolus victoria, the causal agent of Victoria blight, is pathogenic due to its production of a toxin called victorin. Victorin sensitivity in oats, barley, Brachypodium spp., and Arabidopsis has been associated with nucleotide-binding site leucine-rich repeat (NLR) genes, a class of genes known for conferring disease resistance. In this work, we investigated the sensitivity of Phaseolus vulgaris to victorin. We found that victorin sensivity in Phaseolus vulgaris is a developmentally regulated, quantitative trait. A single quantitative trait locus (QTL) accounted for $34 \%$ of the phenotypic variability in victorin sensitivity among Stampede $\times$ Red Hawk $(S \times R)$ recombinant inbred lines. We cloned two NLR-encoding genes within this QTL and showed one, Phvul05G031200 (PvLOV), confers victorin-dependent cell death when overexpressed in Nicotiana benthamiana. Protein sequences of PvLOV from victorin-sensitive and the victorinresistant bean parents differ by two amino acids in the leucinerich repeat region, but both proteins confer victorin-dependent cell death when overexpressed in $N$. benthamiana.
\end{abstract}

Breeding disease resistant plants is an environmentally friendly strategy for preventing crop loss due to disease. However, studies of Victoria blight suggest that selection for resistance to one disease can inadvertently select for susceptibility to another. Cochliobolus victoriae is a necrotrophic fungus that causes Victoria blight on oats and innocuous infection on several weedy species, soybean, and barley (Meehan and Murphey 1946, 1947). In the 1940s, oat genotypes derived from the Victoria cultivar and widely planted as a source of resistance to the crown rust fungus Puccinia coronata were also susceptible to $C$. victoriae, and a Victoria blight epidemic ensued. The epidemic was the result of tight genetic linkage between a dominant susceptibility gene to Victoria blight, $V b$, and the dominant Puccinia coronata resistance gene Pc-2 (Welsh

${ }^{\dagger}$ Corresponding author: T. J. Wolpert;
E-mail: wolpertt@science.oregonstate.edu

Funding: This project was supported by the Agriculture and Food Research Initiative Competitive Grants Program through the United States Department of Agriculture National Institute of Food and Agriculture grant numbers 2012-67013-19291 and 2016-67013-24736.

*The $\boldsymbol{e}$-Xtra logo stands for "electronic extra" and indicates that six supplementary figures and one supplementary table are published online.

(c) 2018 The American Phytopathological Society et al. 1954). $V b$ confers susceptibility by conditioning sensitivity to the $C$. victoriae toxin victorin, an essential nectrophic effector. The possible identity of $V b$ and $P c$-2 suggests victorin incites a resistance-associated type of cell death; a notion corroborated by investigations in Arabidopsis, sorghum, and wheat, in which necrotrophic pathogens co-opted likely resistance responses to cause disease (Faris et al. 2010; Lorang et al. 2007, 2012; Nagy and Bennetzen 2008; Stukenbrock and McDonald 2009; Wolpert et al. 2002).

In studies of common bean (Phaseolus vulgaris L.), Wheeler and Pirone (1969) reported that plants treated with crude preparations of victorin became virus-resistant and, at higher concentrations, developed necrosis. Necrosis was assumed to be nonspecific. In this work, we investigated the genetic basis of possible victorin sensitivity in Phaseolus vulgaris. We quantified victorin sensitivity in a Stampede (victorin-sensitive) $\times$ Red Hawk (victorin-insensitive) recombinant inbred population (Song et al. 2015) and identified a quantitative trait locus (QTL) that contains two nucleotide-binding site, leucine-rich-repeat (NLR)encoding genes, one of which confers victorin sensitivity when expressed in Nicotiana benthamiana.

\section{RESULTS}

We screened common bean germplasm for sensitivity to victorin and observed mild to severe victorin-induced necrosis on most Phasolus vulgaris lines at the adult stage. The pinto marketclass bean Stampede displays strong sensitivity to victorin and the dark red kidney bean Red Hawk is nearly insensitive to victorin (Fig. 1). Because a genetic map derived from $267 \mathrm{~F} 2 \mathrm{~s}$ of a Stampede $\times$ Red Hawk $(\mathrm{S} \times \mathrm{R})$ mapping population is available (Song et al. 2015), we chose to screen a $S \times R$ F5:6 mapping population of 164 recombinant inbred lines (RILs) to investigate the genetic basis of victorin sensitivity in common bean.

We observed that Stampede plants treated with victorin display a developmentally regulated response. Young Stampede seedlings are nearly insensitive to victorin, but at approximately 3 weeks after germination, when the third trifoliate leaf emerges, the plant becomes sensitive to a concentration of $20 \mu \mathrm{g} / \mathrm{ml}$ victorin toxin (Fig. 1). At this stage all leaves of a sensitive plant display sensitivity that typically increases with plant age. We assigned a cumulative score ranging from 0 (insensitive) to 3 (strong sensitivity) to each of the 164 RILs based on the degree of visible necrosis over time (Supplementary Fig. S1). 
Mapping, quantitative and qualitative genetic analysis.

Phenotypic analyses of the 164 RILs suggest victorin sensitivity is a genetically complex, quantitative trait in the $\mathrm{S} \times \mathrm{R}$ population. A total of 133 lines (81\%) scored as sensitive to victorin (31\% strong [score 3], 22\% moderate [score 2], $28 \%$ mild [score $1]$ ), indicating that multiple loci condition sensitivity to victorin in this population (Supplementary Table S1). A high-resolution genetic linkage map was generated using the R/qtl software package (Broman et al. 2003). Phenotypic data were also analyzed in R/qtl using the Haley-Knot regression method (Haley and Knott 1992). Significance of the peak $\log$ of the likelihood (LOD) score was assessed using 1,000 permutations of the data and a $5 \%$ experiment-wide error rate to obtain a significance LOD threshold of 3.4. The 1.5 LOD support interval for the location of the identified QTL was calculated using the lodint function. A major QTL (PvLOV5.1) was identified on chromosome Pv05, centered at 29.0 centimorgans $(\mathrm{cM})$ with a peak LOD of 14.9. The 1.5 support interval $(27.2$ to $32.0 \mathrm{cM})$ explained $34 \%$ of the phenotypic variability (Fig. 2).

Qualitative analysis of RIL subpopulations consisting of discrete phenotypic or combined phenotypic groupings was performed in an effort to parse out contributions of individual loci to quantitative victorin-sensitivity phenotypes. For

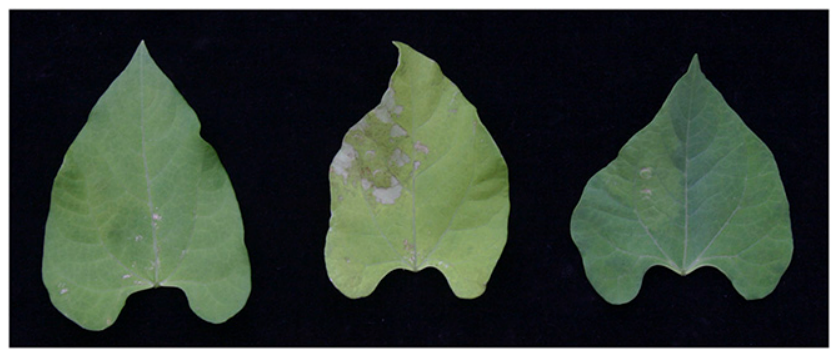

Fig. 1. Leaves of three-week-old Red Hawk (left) or Stampede (middle), and 10-day-old Stampede (right), three days after infiltration, left of the midvein, with $20 \mu \mathrm{g}$ of victorin per milliliter. example, combining scores for strong and moderate sensitivity ( $3[n=51]$ and $2[n=36]$, respectively) and those for mild or no sensitivity $(1[n=46]$ and $0[n=31]$, respectively) resulted in a ratio of sensitive to insensitive plants of $87: 77$. This ratio conforms to a $1: 1$ ratio $\left(x^{2}=0.61,1\right.$ degree of freedom; twotailed $P=0.43$ ) as expected for a single dominant gene. However, reanalysis of the phenotype as a binary trait significantly lowered the peak LOD of the Pv05 QTL, and thus, we reject the hypothesis that only one gene accounts for the phenotypes observed. Goodness-of-fit for data conforming to a model with two independent, complementary genes $($ ratio $=3$ : 1 , rating 0 versus rating $1+2+3)$ could not be rejected $\left(x^{2}=\right.$ $3.252,1$ degree of freedom; two-tailed $P=0.0713$ ). However, contributions of only two genes is hard to reconcile with the quantitative sensitivity phenotypes observed. Qualitative analysis of subpopulations containing individual victorin-sensitivity phenotypes and insensitive plants (e.g., scores 0 and 1,0 and 2, or 0 and 3) significantly increased the 1.5 LOD of the Pv05 QTL from 14.93 to 25.40 and narrowed the interval of this peak from 4.8 to $2.0 \mathrm{cM}$ (28.2 to $30.2 \mathrm{cM})$ when only strongly sensitive and insensitive subpopulations (scores 3 and 0 ) were considered (Supplementary Fig. S2). The QTL LOD score did not increase when mild or moderately sensitive phenotypes and insensitive phenotypes (scores of 1 or 2 versus 0 ) were considered, either together or independently. Significantly, the $2.0 \mathrm{cM}$, Pv05 interval contains only 27 genes, including two NLR genes (Fig. 3). There were no genetic recombinants between the NLR genes (Phvul05G030500 and Phvul05G031200) and the victorinsensitivity phenotype when strongly sensitive and insensitive subpopulations (scores 3 and 0 ) were considered.

\section{Identification of $P v L O V$.}

We cloned both Phvul05G030500 and Phvul05G031200 from Stampede, the victorin-sensitive parent, and transiently overexpressed them in leaves of $N$. benthamiana. We found Phvul05G031200 but not Phvul05G030500 conferred victorindependent cell death in $N$. benthamiana (Fig. 4). We therefore designate Phvul05G031200 as PvLOV (for locus orchestrating

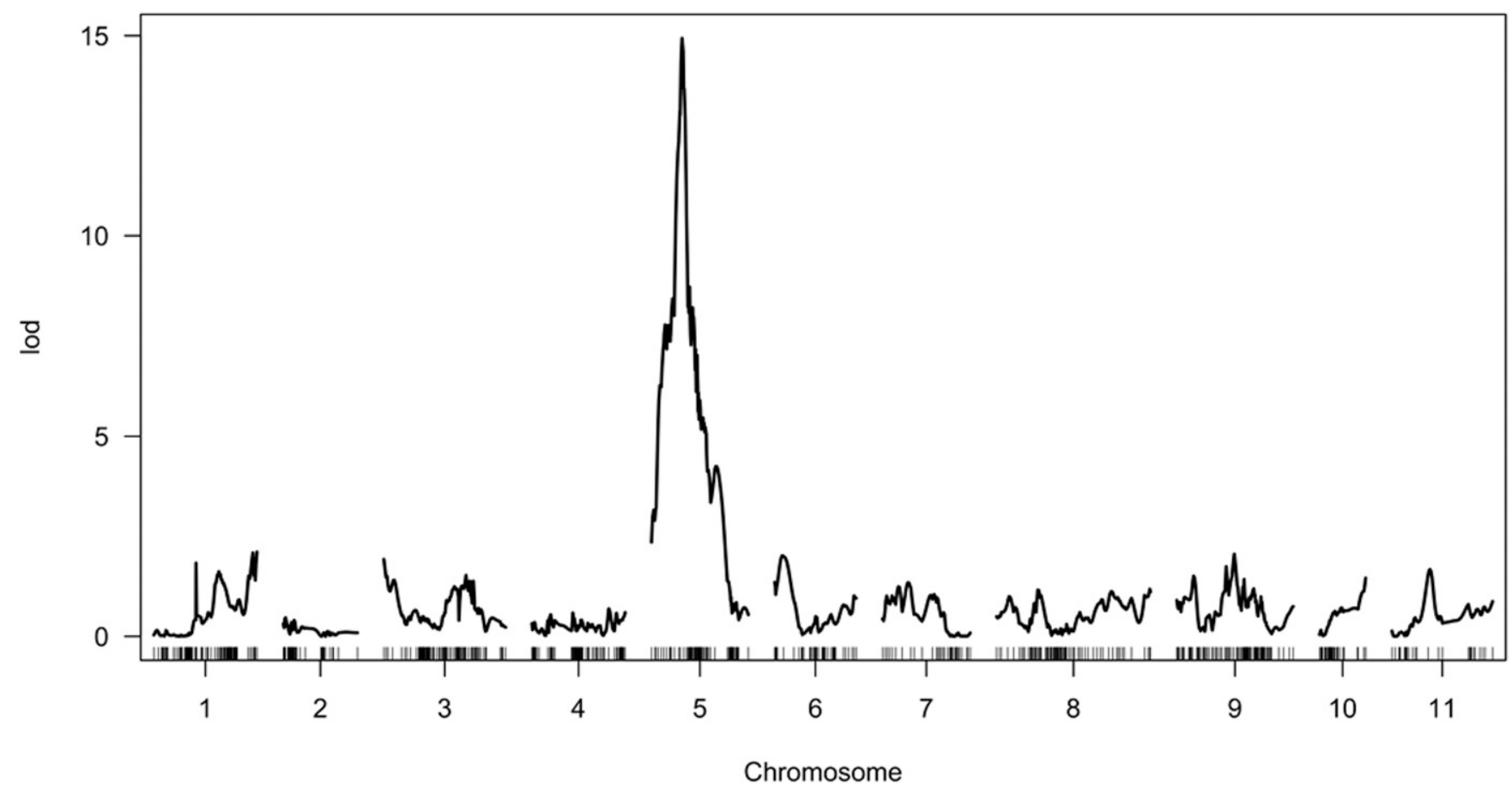

Fig. 2. Interval mapping of victorin sensitivity in bean showing a major quantitative trait locus centered at 29.0 centimorgans on chromosome Pv05 with a peak $\log$ of the likelihood (LOD) score of 14.93 . 
victorin effects). We sequenced $P v L O V$ and determined the inferred protein sequences of PvLOV from the victorin-sensitive (Stampede) and the victorin-insensitive (Red Hawk) parents differ by two amino acids in the leucine-rich repeat region (Supplementary Fig. S3). This finding is corroborated by common bean sequences available at the National Center for Biotechnology Information (NCBI) (accession number PRJNA 386820). Interestingly, the reference genome ecotype G19833 (Schmutz et al. 2014) shares the Red Hawk PvLOV allele but is sensitive to victorin, thus confirming the idea that victorin sensitivity is a complex, quantitative trait and that loci in addition to $P v L O V$ can contribute to victorin sensitivity.

Surprisingly both $P v L O V \mathrm{~S}$ and R confer victorin-dependent cell death when transiently overexpressed in leaves of $N$. benthamiana, while neither gene induces cell death in the absence of victorin (Supplementary Fig. S4).

Because both $P v L O V \mathrm{~S}$ and $\mathrm{R}$ confer victorin-dependent cell death when transiently overexpressed in leaves of $N$. benthamiana, we investigated the possibility that expression levels of $P v L O V$ in $\mathrm{S}$ and $\mathrm{R}$ account for the differential victorin sensitivity of these cultivars. However, we found expression levels of $P v L O V$ in Stampede and Red Hawk leaves do not significantly differ at 3 to 4 weeks of age, when Stampede is sensitive to victorin but Red Hawk is not, nor was $P v L O V$ expression induced by treatment with victorin (Supplementary Fig S5). We therefore assume that sequence differences in the leucine-rich repeat region of the proteins must account for the differential phenotypes they confer in Stampede and Red Hawk, even though the proteins do not confer differential phenotypes when overexpressed in $N$. benthamiana.

\section{cM}

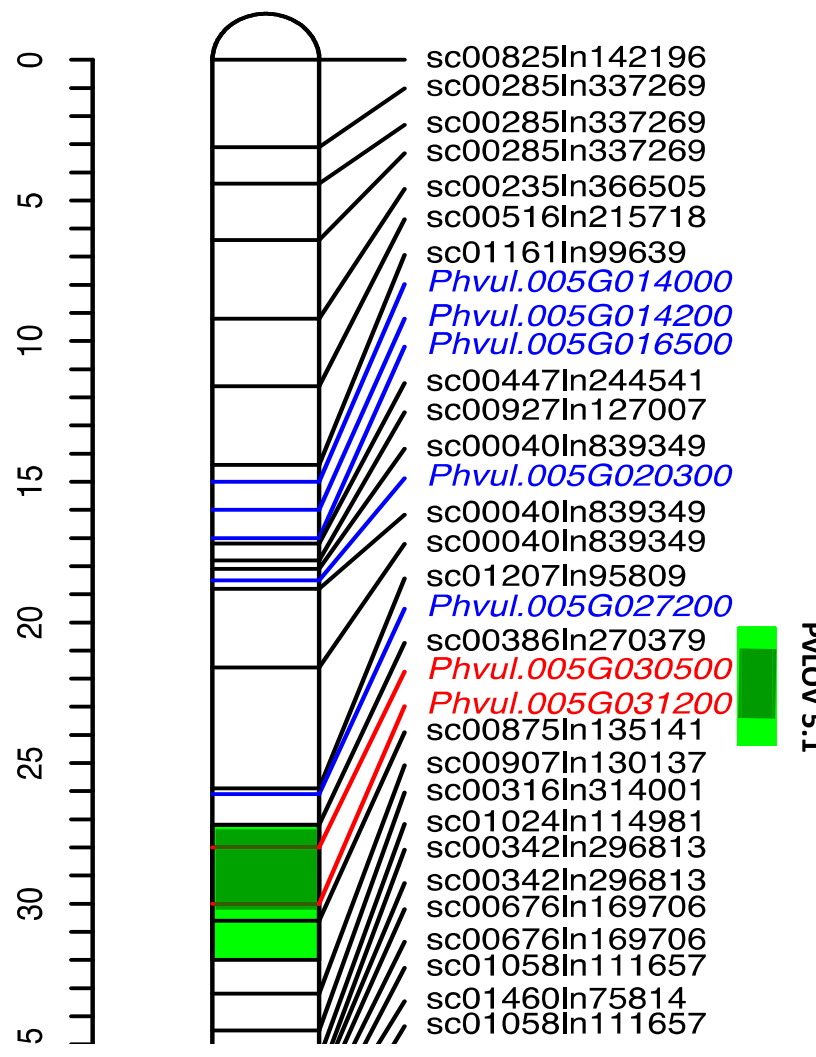

Fig. 3. Map of Phaseolus vulgaris chromosome Pv05, indicating the relative location of annotated nucleotide-binding site leucine-rich repeat genes (blue or red) to molecular markers (black) and the victorin-sensitivity quantitative trait locus PvLOV 5.1 (green rectangle, darker green denotes a 2-centimorgan (cM) binary trait interval).
Interestingly, $P v L O V$ does not confer victorin-dependent cell death when overexpressed in Arabidopsis. We created 16 35S: $P v L O V$ transgenic Col-0 Arabidopsis lines and assayed them for victorin sensitivity in the T1 to T3 generations but none displayed victorin sensitivity (not shown). PvLOV shares only $28 \%$ amino acid identity with AtLOV, a NLR that confers victorin sensitivity in Arabidopsis (Lorang et al. 2007) AtLOV is not among the most closely related Arabidopsis genes to $P v L O V$, and $P v L O V$ is not among the most closely related P. vulgaris genes to AtLOV by BLST BLAST (Basic Local Alignment Search Tool) analysis. In a study on comparative genomics of non-TNL resistance genes from six species including Arabidopsis and P. vulgaris, AtLOV falls into the CNL-D grouping and $P v L O V$ most closely aligns to the CNL-C grouping (Nepal et al. 2017).

Neither Stampede nor Red Hawk display significant susceptibility to $C$. victoriae. Colonization of leaf tissue by the fungus occurs on both genotypes only saprophytically once leaves begin to senesce (not shown). We postulate that germinating spores do not produce sufficient toxin on green leaves of each parental genotype to overcome the basal resistance that prevents infection of bean. The concentration of victorin required to consistently induce cell death in $P$. vulgaris $(20 \mu \mathrm{g} / \mathrm{ml})$ is approximately 1,000-fold more than that required for oat (Wolpert et al. 1988) (Supplementary Fig. S6).

\section{DISCUSSION}

Disease resistance genes can impart a fitness cost to plants (Brown 2016; Orgil et al. 2007; Tian et al. 2003). One cost is exemplified in the growing body of work suggesting some necrotrophic pathogens exploit NLR proteins, a class of proteins typically associated with resistance, to cause rather than

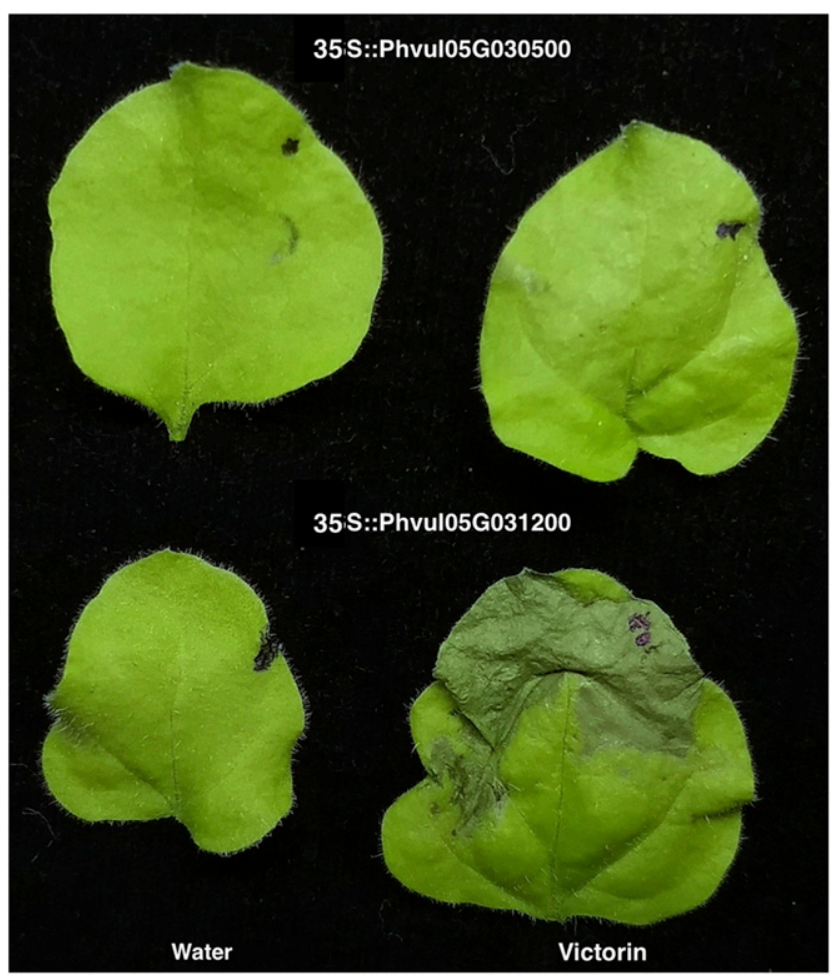

Fig. 4. Nicotiana benthamiana leaves transiently expressing Phvul05G031200 or Phvul05G030500 with the Cauliflower mosaic virus $35 \mathrm{~S}$ promoter in pEarleygate $100,24 \mathrm{~h}$ after infiltration with $10 \mu \mathrm{g}$ of victorin per milliliter or $\mathrm{H}_{2} \mathrm{O}$. All five replicate leaves per treatment gave equivalent results and the experiment was repeated twice. 
curtail disease (Faris et al. 2010; Lorang et al. 2007, 2012; Nagy and Bennetzen 2008; Stukenbrock and McDonald 2009; Wolpert et al. 2002). Previously, we reported that victorin sensitivity and susceptibility to $C$. victoriae in Arabidopsis depend on activation of an NLR protein according to the guard model of plant defense (Lorang et al. 2012) and victorin sensitivity of barley maps to a resistance gene-rich region (Lorang et al. 2010). Compelled by these findings and an older report that functional resistance to viruses in beans can be induced by treatment with victorin (Wheeler and Pirone 1969), we chose to investigate the genetic basis of victorin sensitivity in $P$. vulgaris. We found that victorin sensitivity in common bean is quantitative and developmentally regulated (Fig. 1). We mapped a major QTL accounting for $34 \%$ of the variability for victorin sensitivity in common bean to a narrow genetic region on chromosome Pv05 that contains two NLR genes (Meziadi et al. 2016) (Fig. 3), the most common class of disease resistance genes. We cloned and sequenced these NLR genes. Phvul05G031200, which we designate $P v L O V$, conferred victorin-dependent cell death when transiently expressed in leaves of $N$. benthamiana (Fig. 4). These data extend the observation of similar occurrences in grasses and Arabidopsis to include a legume.

Our finding that victorin sensitivity in common bean is phenotypically quantitative and maps to one major QTL accounting for $34 \%$ of the phenotypic variability has interesting implications. The remaining, unresolved phenotypic variability constitutes a genetic background (GB) effect, by definition. A GB effect is further demonstrated by our findng that $P v L O V$ conditions victorin-dependent cell death in $N$. benthamiana but not in Arabidopsis. Furthermore, the P. vulgaris reference genome ecotype G19833 (Schmutz et al. 2014) is among only 17 of 188 sequenced Durango genotypes (NCBI BioProject database) that has the Red Hawk allele for $P v L O V$ but, like many common beans, is still sensitive to victorin. Unresolved factors that contribute to the victorin-sensitivity phenotype must be either positive acting, such as observed for multiple resistance (R) gene loci (Barbary et al. 2016), negative acting, such as factors that differentially temper cell death conditioned by Red Hawk (G19833) and Stampede alleles of PvLOV, or likely, a combination of both factors (Iakovidis et al. 2016). In the former case, multiple, positive-acting genes could result from redundancy in NLR proteins "guarding" a key cellular target of victorin or multiple cellular targets for victorin guarded by multiple resistance proteins. H-type thioredoxins (TRXh) are known cellular targets of victorin in Arabidopsis (Lorang et al. 2012; Sweat and Wolpert 2007). P. vulgaris has at least ten TRXh-like proteins (Phytozome database) (Schmutz et al. 2014). Whether these thioredoxins are involved in defense (as in Arabidopsis) and are cellular targets of a bean pathogen effector has not been investigated and is challenging to address because $P$. vulgaris is recalcitrant to transformation (HnatuszkoKonka et al. 2014). Factors that could negatively regulate victorin sensitivity, on the other hand, such as those controlling physiology or development that temper cell death (Bruggeman et al. 2015; Huysmans et al. 2017) would not only reduce the extent of NLR-mediated cell death induced by the necrotophic effector victorin but could simultaneously reduce the cost of maintaining NLR genes that provide a resistance function. In any scenario, GB effects have potential to influence both biotrophic and necrotrophic disease outcomes, and GB selected to enhance biotrophic disease resistance could inadvertently increase nocrotrophic susceptibility.

Here, we report that sensitivity to victorin is mild and developmentally regulated for $P$. vulgaris, much like what has been observed for Arabidopsis, barley, Brachypodium spp., and rice (Lorang et al. 2012) and in contrast to strong oat sensitivity. Because victorin sensitivity has clearly been associated with
NLR-encoding genes, developmental sensitivity to victorin is reflective of developmental disease resistance. Developmental, age-related resistance (ARR) has been observed widely, for both single-gene (Carrella et al. 2015) and quantitative resistance (Shah et al. 2015), but its mechanisms and the selection pressures for its evolution, in most cases, remain undefined (Develey-Rivière and Galiana 2007; Wilson et al. 2017). Developmental changes in $P v L O V$ gene expression were not significant (Supplementary Fig. 5S) and, therefore, do not explain age-related victorin sensitivity, just as ARR is not attributed to developmental changes in resistance gene expression in most cases studied (Develey-Rivière and Galiana 2007; Wilson et al. 2017). Juvenile sensitivity to victorin, on the other hand, contributed to severe disease outbreaks on oats because infected seedlings typically died (Meehan and Murphy 1946). Thus, where an NLR gene conferring juvenile disease resistance would likely be selected for in the presence of a formidable, cognate biotrophic pathogen, the same gene would be quickly selected against if it conferred juvenile susceptibility to a resident necrotroph. For this reason, the idea that developmental resistance may represent a compromise in selection for NLR genes that simultaneously afford adult immunity to biotrophs or hemibiotrophs while protecting seedlings from necrotrophs like C. victoriae is appealing. Whether necrotrophic pathogens have had a role in the evolution of developmental disease resistance is an interesting question that is not easily addressed. The fact that developmental sensitivity to victorin does not translate to significant pathogenicity of $C$. victoriae on bean or barley suggests evolution of a developmental phenotype may have been advantageous in these cases.

In summary, we have shown that phenotypic expression of victorin sensitivity in common bean, as previously observed in barley, is subject to developmental stage and genetic background effects which, by definition, we could not define. The genetic contribution to victorin sensitivity that we defined as a major chromosome Pv05 QTL correlates with the presence of a gene encoding a NLR protein, one which we have shown to condition victoirn-dependent cell death (Fig. 4). If victorin sensitivity is indeed conditioned by genes typically associated with disease resistance in diverse plant families (Poaceae [Meehan and Murphy 1947; Lorang et al. 2010], Brassicaceae [Lorang et al. 2004], and Fabaceae [this work]), then these $R$ genes are also poised to act as potential susceptibility genes when introduced into an accommodating genetic context and in the presence of the pathogen. This likely occurred for C. victoriae and oat in the 1940s and the resultant Victoria blight epidemics (Wolpert et al. 2002). For this reason, identifying which NLR genes have the potential to condition susceptibility to necrotrophic pathogens is of interest.

\section{MATERIALS AND METHODS}

Plant material and victorin assays.

Seed of 164 Redhawk $\times$ Stampede F5:F6 RILs (Song et al. 2015) were provided by J. Osorno (North Dakota State University). Seeds were sown in 1-gallon pots of LA4PC soil mix (Sungrow) and were grown at $24^{\circ} \mathrm{C}$ with an approximate $12-\mathrm{h}$ light, 12-h dark cycle in the greenhouse. Plants were assayed for victorin sensitivity at regular intervals from three weeks of age until flowering, by infiltrating successive leaves with $20 \mu \mathrm{g}$ of victorin per milliliter. A score of 0 to $3(0=$ no visible necrosis, $1=$ necrosis in up to $20 \%$ infiltrated area, $2=$ necrosis in approximately $50 \%$ infiltrated area, $3=$ necrosis in all infiltrated regions by $48 \mathrm{~h}$ after infiltration) was assigned to each RIL, based on the evaluation of four plants per RIL. Experiments were repeated three times. 


\section{QTL analysis.}

QTL analysis was performed in $\mathrm{R}$ with the software package R/qtl (Broman et al. 2003). Phenotypic data were also analyzed in R/qtl, using the Haley-Knot regression method (Haley and Knott 1992). Significance of the peak LOD score was assessed using 1,000 permutations of the data and a 5\% experiment-wide error rate. The 1.5 LOD support interval for the location of the identified QTL was calculated using the lodint function.

\section{DNA isolation, polymerase chain reaction (PCR), and cloning.}

Genomic DNA of $P$. vulgaris was isolated using the DNeasy plant minikit (Qiagen). The primers Pdon31200F: CAAAAAAGC AGGCTCCATGGCAGCAGAGTTGATAGGT and Pdon31200R: GAAAGCTGGGTGCTAATAAATCATAACAGAAGGGAT and Pdon30500F: CAAAAAAGCAGGCTCCATGGAGCAACCTT CTTCCATG and Pdon30500R: GAAAGCTGGGTGTCATGG AATCCAAAACTTCCT were designed to amplify genomic clones of Phvul05G031200 and Phvul05G030500, respectively, from the start to the stop codons, using PCR according to standard protocols. The resulting PCR products were first cloned into the entry vector pDON207 (Invitrogen) and were then recombined into the binary vector pEarlygate100, which utilizes the Cauliflower mosaic virus 35S promoter (Earley et al. 2006), using Gateway technology (Invitrogen). The resulting plasmids were electroporated into Agrobacterium tumefaciens GV2260 for transient expression in $N$. benthamiana and strain GV3101 for transformation of Arabidopsis.

\section{Plant transformations.}

For transient expression in N. benthamiana, Agrobacterium tumefaciens GV2260 containing the pEarlygate 100 constructs described above were grown in King's B medium overnight at $30^{\circ} \mathrm{C}$. Cells were pelleted and resuspended in infiltration solution $(10 \mathrm{mM} \mathrm{MgCl}$ and $10 \mathrm{mM}$ MES buffer, $\mathrm{pH} 5.3,1 \mathrm{mM}$ acetosyringone) to an optical density at $600 \mathrm{~nm}$ of 0.5 and incubated at room temperature for $2 \mathrm{~h}$, before being syringeinfiltrated into the youngest fully expanded leaf of threeweek-old $N$. benthamiana. For victorin-sensitivity assays, victorin or water was infiltrated $48 \mathrm{~h}$ after Agrobacterium tumefaciens infiltration. Pictures were taken $24 \mathrm{~h}$ after victorin treatment.

Agrobacterium tumefaciens GV3101 harboring the Pearlygate 100 constructs described above were transformed into Arabidopsis (Col-0) by the floral-dip method (Clough and Bent 1998) T0 seed from dipped plants were grown on soil and transformed plants were selected using Finale herbicide (Bayer). The presence of transgenes was confirmed by PCR analysis. Transgenic plants were screened for victorin sensitivity in the $\mathrm{T} 1$ to $\mathrm{T} 3$ generations by infiltrating $10 \mu \mathrm{g}$ of victorin per milliliter into the third to fifth leaves of 4 -week-old plants.

\section{Quantitative (q)PCR.}

RNA was isolated from trifoliate leaves of 4-week-old $P$. vulagaris because, at this stage, leaves of Stampede are sensitive to victorin but leaves of Red Hawk are not. RNA was isolated using the Aurum Total RNA minikit (Bio-Rad). cDNA was made using the iScript cDNA synthesis kit and qPCR was performed using iQSYBR green supermix (both from Bio-Rad) on a Bio-Rad CFX100 real-time thermocycler, according to the manufacturer's recommendations. Data are based on an $n$ value $=6$, for three biological replications $\times$ two technical replications. qPCR primers were designed using Primer3 (Untergasser et al. 2012) and expression was normalized to the $P$. vulgaris $I D E$ (insulin degrading enzyme) reference gene (Borges et al. 2012). Data were analyzed with the $\Delta \Delta \mathrm{qC}$ method using CFX Manager (3.1) software (Bio-Rad).

\section{ACKNOWLEDGMENTS}

Thank you to J. Osorno for seed of Stampede $\times$ Redhawk recombinant inbred lines.

\section{LITERATURE CITED}

Barbary, A., Djian-Caporalino, C., Marteu, N., Fazari, A., Caromel, B., Castagnone-Sereno, P., and Palloix, A. 2016. Plant genetic background increasing the efficiency and durability of major resistance genes to root-knot nematodes can be resolved into a few resistance QTLs. Front. Plant Sci. 7:632.

Borges, A., Tsai, S. M., and Caldas, D. G. 2012. Validation of reference genes for RT-qPCR normalization in common bean during biotic and abiotic stresses. Plant Cell Rep. 31:827-838.

Broman, K. W., Wu, H., Sen, S., and Churchill, G. A. 2003. R/qtl: QTL mapping in experimental crosses. Bioinformatics 19:889-890.

Brown, J. K. M. 2016. Fitness costs of pathogen recognition in plants and their implications for crop improvement. Pages 385-404 in: Plant Pathogen Resistance Biotechnology. D. B. Collinge, ed. John Wiley \& Sons, Hoboken, NJ, U.S.A.

Bruggeman, Q., Raynaud, C., Benhamed, M., and Delarue, M. 2015. To die or not to die? Lessons from lesion mimic mutants. Front. Plant Sci. 6:24.

Carella, P., Wilson, D. C., and Cameron, R. K. 2015. Some things get better with age: Differences in salicylic acid accumulation and defense signaling in young and mature Arabidopsis. Front. Plant Sci. 5:775.

Clough, S. J., and Bent, A. F. 1998. Floral dip: A simplified method for Agrobacterium-mediated transformation of Arabidopsis thaliana. Plant J. 16:735-743.

Develey-Rivière, M. P., and Galiana, E. 2007. Resistance to pathogens and host developmental stage: A multifaceted relationship within the plant kingdom. New Phytol. 175:405-416.

Earley, K. W., Haag, J. R., Pontes, O., Opper, K., Juehne, T., Song, K., and Pikaard, C. S. 2006. Gateway-compatible vectors for plant functional genomics and proteomics. Plant J. 45:616-629.

Faris, J. D., Zhang, Z., Lu, H., Lu, S., Reddy, L., Cloutier, S., Fellers, J. P., Meinhardt, S. W., Rasmussen, J. B., Xu, S. S., Oliver, R. P., Simons, K. J., and Friesen, T. L. 2010. A unique wheat disease resistance-like gene governs effector-triggered susceptibility to necrotrophic pathogens. Proc. Natl. Acad. Sci. U.S.A. 107:13544-13549.

Haley, C. S., and Knott, S. A. 1992. A simple regression method for mapping quantitative trait loci in line crosses using flanking markers Hered. Edinb 69:315-324.

Hnatuszko-Konka, K., Kowalczyk, T., Gerszberg, A., Wiktorek-Smagur, A., and Kononowicz, A. K. 2014. Phaseolus vulgaris-Recalcitrant potential. Biotechnol. Adv. 32:1205-1215

Huysmans, M., Lema, S. A., Coll, N. S., and Nowack, M. K. 2017. Dying two deaths-Programmed cell death regulation in development and disease. Curr. Opin. Plant Biol. 35:37-44.

Iakovidis, M., Teixeira, P. J. P. L., Exposito-Alonso, M., Cowper, M. G., Law, T. F., Liu, Q., Vu, M. C., Dang, T. M., Corwin, J. A., Weigel, D., Dangl, J. L., and Grant, S. R. 2016. Effector-triggered immune response in Arabidopsis thaliana is a quantitative trait. Genetics 204:337-353.

Lorang, J., Kidarsa, T., Bradford, C. S., II, Gilbert, B., Curtis, M., Tzeng, S. C., Maier, C. S., and Wolpert, T. J. 2012. Tricking the guard: Exploiting plant defense for disease susceptibility. Science 338:659-662

Lorang, J. M., Carkaci-Salli, N., and Wolpert, T. J. 2004. Identification and characterization of victorin sensitivity in Arabidopsis thaliana. Mol. Plant-Microbe Interact. 17:577-582.

Lorang, J. M., Cuesta-Marcos, A., Hayes, P. M., and Wolpert, T. J. 2010 Identification and mapping of adult-onset sensitivity to victorin in barley. Mol. Breed. 26:545-550.

Lorang, J. M., Sweat, T. A., and Wolpert, T. J. 2007. Plant disease susceptibility conferred by a "resistance" gene. Proc. Natl. Acad. Sci. U.S.A. 104:14861-14866.

Meehan, F., and Murphy, H. C. 1946. A new Helminthosporium blight of oats. Science 104:413-414.

Meehan, F., and Murphy, H. C. 1947. Differential phytotoxicity of metabolic byproducts of Helminthosporium victoriae. Science 106:270-271.

Meziadi, C., Richard, M. M. S., Derquennes, A., Thareau, V., Blanchet, S., Gratias, A., Pflieger, S., and Geffroy, V. 2016. Development of molecular markers linked to disease resistance genes in common bean based on whole genome sequence. Plant Sci. 242:351-357.

Nagy, E. D., and Bennetzen, J. L. 2008. Pathogen corruption and site-directed recombination at a plant disease resistance gene cluster. Genome Res. 18: 1918-1923.

Nepal, M. P., Andersen, E. J., Neupane, S., and Benson, B. V. 2017. Comparative genomics of non-TNL disease resistance genes from six plant species. Genes (Basel) 8:249. 
Orgil, U., Araki, H., Tangchaiburana, S., Berkey, R., and Xiao, S. 2007. Intraspecific genetic variations, fitness cost and benefit of $R P W 8$, a disease resistance locus in Arabidopsis thaliana. Genetics 176:2317-2333.

Schmutz, J., McClean, P. E., Mamidi, S., Wu, G. A., Cannon, S. B., Grimwood, J., Jenkins, J., Shu, S., Song, Q., Chavarro, C., Torres-Torres, M., Geffroy, V., Moghaddam, S. M., Gao, D., Abernathy, B., Barry, K., Blair, M., Brick, M. A., Chovatia, M., Gepts, P., Goodstein, D. M., Gonzales, M., Hellsten, U., Hyten, D. L., Jia, G., Kelly, J. D., Kudrna, D., Lee, R., Richard, M. M., Miklas, P. N., Osorno, J. M., Rodrigues, J., Thareau, V., Urrea, C. A., Wang, M., Yu, Y., Zhang, M., Wing, R. A., Cregan, P. B., Rokhsar, D. S., and Jackson, S. A. 2014. A reference genome for common bean and genome-wide analysis of dual domestications. Nat. Genet. 46:707-713.

Shah, S. R. A., Tao, L., Haijuan, C., Lei, L., Zheng, Z., Junming, L., and Yongchen, D. 2015. Age-related resistance and the defense signaling pathway of Ph-3 gene against Phytophthora infestans in tomatoes. Hortic. Plant J. 1:70-76.

Song, Q., Jia, G., Hyten, D. L., Jenkins, J., Hwang, E. Y., Schroeder, S. G., Osorno, J. M., Schmutz, J., Jackson, S. A., McClean, P. E., and Cregan, P. B. 2015. SNP assay development for linkage map construction, anchoring whole-genome sequence, and other genetic and genomic applications in common bean. G3 (Bethesda) 5:2285-2290.

Stukenbrock, E. H., and McDonald, B. A. 2009. Population genetics of fungal and oomycete effectors involved in gene-for-gene interactions. Mol. Plant-Microbe Interact. 22:371-380.

Sweat, T. A., and Wolpert, T. J. 2007. Thioredoxin $h 5$ is required for victorin sensitivity mediated by a CC-NBS-LRR gene in Arabidopsis. Plant Cell 19:673-687.

Tian, D., Traw, M. B., Chen, J. Q., Kreitman, M., and Bergelson, J. 2003. Fitness costs of R-gene-mediated resistance in Arabidopsis thaliana. Nature 423:74-77.
Untergasser, A., Cutcutache, I., Koressaar, T., Ye, J., Faircloth, B. C., Remm, M., and Rozen, S. G. 2012. Primer3-New capabilities and interfaces. Nucleic Acids Res. 40:e115.

Welsh, J. N., Peturson, B., and Machacek, V. 1954. Associated inheritance of reaction to races of crown rust, Puccinia coronata avenae Erikss., and to Victoria blight, Helminthosporium victoriae M. and M., in oats. Can. J. Bot. 32:55-68.

Wheeler, H., and Pirone, T. P. 1969. Pathotoxin-induced disease resistance in plants. Science 166:1415-1417.

Wilson, D. C. X., Kempthorne, C. J., Carella, P., Liscombe, D. K., and Cameron, R. K. 2017. Age-related resistance in Arabidopsis thaliana xinvolves the MADS-domain transcription factor SHORT VEGETATIVE PHASE and direct action of salicylic acid on Pseudomonas syringae. Mol. Plant-Microbe Interact. 30:919-929.

Wolpert, T. J., Dunkle, L. D., and Ciuffetti, L. M. 2002. Host-selective toxins and avirulence determinants: What's in a name? Annu. Rev. Phytopathol. 40:251-285.

Wolpert, T. J., Macko, V., Acklin, W., and Arigoni, D. 1988. Molecular features affecting the biological activity of the host-selective toxins from Cochliobolus victoriae. Plant Physiol. 88:37-41.

\section{AUTHOR-RECOMMENDED INTERNET RESOURCES}

The Basic Local Alignment Search Tool: blast.ncbi.nlm.nih.gov Department of Energy Joint Genome Institute Phytozome database: https://phytozome.jgi.doe.gov

LinkageMapView database: https://cran.r-project.org/web/packages/LinkageMapView/index.html

NCBI's BioProject Phaseolus vulgaris database: https://www.ncbi.nlm.nih.gov/bioproject/?term=PRJNA386820 